\title{
Referral criteria for school-based hearing screening in South Africa: Considerations for resource-limited contexts
}

\author{
Faheema Mahomed-Asmail $^{a, *}$, De Wet Swanepoel ${ }^{a, b, c}$, \\ Robert H. Eikelboom ${ }^{a, b, c}$ \\ a Department of Speech-Language Pathology and Audiology, University of Pretoria, Pretoria, South Africa \\ ${ }^{\mathrm{b}}$ Ear Sciences Centre, School of Surgery, The University of Western Australia, Nedlands, Australia \\ ${ }^{c}$ Ear Science Institute Australia, Subiaco, Australia
}

\section{A R T I C L E I N F O}

Article history:

Received 29 January 2015

Accepted 2 November 2015

Available online 8 March 2016

\section{Keywords:}

School hearing screening

Screening protocol

Rescreen

Screening intensity

Referral rate

Cost-effectiveness

\begin{abstract}
A B S T R A C T
Background: School-based hearing screening is likely to be the first opportunity to identify childhood hearing loss in South Africa. Criteria for school-based hearing screening requires balancing the targeted degree of hearing loss while ensuring that referral rates are sufficiently low for a cost-effective and sustainable programme. The study aim was to investigate the effect of screening intensity (loudness) levels on the referral rate and to establish the effect of an immediate rescreen in reducing the referral rate.

Methods: A within-subject study was conducted in two phases. Phase 1: compared the referral rate in a counterbalanced sequence at screening levels of $20 \mathrm{~dB} H \mathrm{HL}, 25 \mathrm{~dB} \mathrm{HL}$ and $30 \mathrm{~dB}$ HL across 1, 2 and $4 \mathrm{kHz}$ for 135 children. Phase 2: determined the effect of an immediate rescreen on referral rate for 337 children screened at $25 \mathrm{~dB}$ HL. If a further referral was obtained on rescreen, diagnostic audiometry was subsequently conducted.

Results: Referral rate was reduced to $6.7 \%$ from $17 \%$ when using $25 \mathrm{~dB} \mathrm{HL}$ as opposed to $20 \mathrm{~dB}$ HL as screening intensity. Referral rate was reduced to $4.4 \%$ when employing $30 \mathrm{~dB}$ $\mathrm{HL}$ as screening intensity. An immediate rescreen reduced the overall referral rate by more than one-third. Diagnostic audiometry confirmed that almost half $(47 \%)$ of the referred children had a hearing loss.

Conclusion: A screening intensity of $25 \mathrm{~dB}$ HL and immediate rescreen reduces the referral rate significantly and will limit the burden of the screening programme on health care resources.

(c) 2015 The Authors. Publishing services by Elsevier B.V. on behalf of Johannesburg University. This is an open access article under the CC BY-NC-ND license (http:// creativecommons.org/licenses/by-nc-nd/4.0/).
\end{abstract}

\footnotetext{
Abbreviations: ESHL, Educationally Significant Hearing Loss; ASHA, American Speech-Language Hearing Association; AAA, American Academy of Audiology; JCIH, Joint Committee of Infant Hearing; NHS, Newborn Hearing Screening.

* Corresponding author.

E-mail addresses: faheema.mahomed@up.ac.za, mahomedfaheema@gmail.com (F. Mahomed-Asmail).

Peer review under responsibility of Johannesburg University.

http://dx.doi.org/10.1016/j.hsag.2015.11.003

1025-9848/@ 2015 The Authors. Publishing services by Elsevier B.V. on behalf of Johannesburg University. This is an open access article under the CC BY-NC-ND license (http://creativecommons.org/licenses/by-nc-nd/4.0/).
} 


\section{Introduction}

Hearing loss is the most common developmental disorder which is identifiable at birth, with an increase in prevalence throughout school-age due to the additions of late-onset, late identified and acquired hearing loss (Fortnum, 2003; Lopez, Mathers, Ezzati, Jamison, \& Murray, 2006; Smith, Bale, \& White, 2005; World Health Organization, 2013). Newborn hearing screening has made early identification of congenital and early-onset hearing loss possible to allow for optimal outcomes through early intervention (Cunningham \& Cox, 2003; Muse et al., 2013). Beyond the newborn period, close to $20 \%$ of permanent, moderate or greater bilateral, mild bilateral and unilateral impairments remain to be identified around the time of school entry due to progressive or delayed-onset hearing loss (American Academy of Audiology (AAA), 2011; Bamford et al., 2007; Grote, 2000).

Nine or ten in every 1000 school-aged children (White, 2010) will potentially have a hearing loss and as a result these students will have difficulties in perceiving speech clearly in social and educational contexts which will contribute to difficulties with attention, learning and social functioning (Bess, Dodd-Murphy 1998; Davis, Elfenbein, Schum, \& Bentler, 1986; McKay, Gravel, \& Tharpe, 2008; World Health Organization, 2013). Minimal and unilateral permanent hearing losses may also result in poorer educational test performance, higher incidence of failed grades and greater dysfunction in areas such as behaviour, energy, stress, social support, self-esteem and socio-emotional aspects (Bess \& Dodd-Murphy, 1998; McKay et al., 2008; Tharpe, 2008).

\subsection{Definition of key concepts}

School-based hearing screening is used to identify children with late-onset or progressive hearing impairments (Meyer, Swanepoel, Van Der Linda, \& Le Roux, 2012; Theunissen \& Swanepoel, 2008). School-based hearing screening is widely recommended (American Speech-Language-Hearing Association (ASHA), 1997; Skarzynski \& Piotrowska, 2012) with clear guidelines in terms of implementation. The universal goal of hearing screening is to identify all children with a significant hearing loss in order to allow for further diagnosis and appropriate intervention (AAA, 2011; ASHA, 1997; Kam et al., 2013; Skarzynski \& Piotrowska, 2012; Theunissen \& Swanepoel, 2008).

School-based hearing screening is of particular importance in countries like South Africa where no legislation or health care mandate is in place to conduct hearing screening on newborns and infants for hearing loss (Meyer et al., 2012; Theunissen \& Swanepoel, 2008). As a result, school-based screening may be the first point of access for detection of hearing loss. The recently launched Integrated School Health Policy (ISHP, 2012) for South Africa acknowledges the importance of hearing screening by including it as part of all the health phases with priority on the foundational phase (Grade R-3). The Integrated School Health Policy (2012) specifies that hearing screening is to be conducted by school health nurses with an audiometer using a screen criteria of $20 \mathrm{~dB} \mathrm{HL}$ intensity at 1, 2 and $4 \mathrm{kHz}$ in accordance with current international guidelines (AAA, 2011; ASHA, 1997).

The pure tone audiometric sweep test has been considered the gold standard and is the most widely used and recommended screening method for school-based hearing screening (AAA, 2011; ASHA, 1997; Bamford et al., 2007). A pure tone signal is presented across different frequencies at a specific screening intensity level; responses to the signals typically include a hand raise or a conditioned response (e.g. dropping a block in a bucket). Although it is easy to administer, successful implementation is often hindered by a number of intrinsic and extrinsic factors. One of these intrinsic factors is to identify the target disorder. For schoolbased hearing screening the target disorder is often referred to as an educationally significant hearing loss (ESHL) (AAA, 2011; ASHA, 1997).

ESHL is considered a hearing loss that interferes with a learner's academic performance (WHO, 2014). This may include permanent sensorineural, conductive and mixed hearing losses, but may also include transient conductive losses. However, the severity of a hearing loss that constitutes ESHL is not always clearly defined. According to the World Health Organisation (2014) a disabling childhood hearing loss constitutes an average hearing threshold in the better ear across the frequencies $0.5,1,2,4 \mathrm{kHz}$ to be $>30 \mathrm{~dB}$ HL. Despite some variability in the frequencies employed for screening, current recommendations generally agree that 1, 2 and $4 \mathrm{kHz}$ should be screened bilaterally (AAA, 2011; ASHA, 1997; ISHP, 2012; Kam et al., 2013; Wu et al., 2014). However, there is less consistency with regards to the screening intensity level that should be used to appropriately identify children with ESHL.

Guidelines specify a screening level of $20 \mathrm{~dB}$ HL across 1,2 or $4 \mathrm{kHz}$ in order to identify an ESHL (American Academy of Audiology, 2011; American Speech-Language-Hearing Association, 1997; Integrated School Health Policy of South Africa, 2012). Despite these guidelines, screening programmes have used various criteria to identify ESHL. For example, Lü et al. (2011) defined a possible hearing loss as an average of $>40 \mathrm{~dB}$ HL across frequencies $(0.5-4 \mathrm{kHz})$ and $\mathrm{Kam}$ et al., (2013) use a screening level of $>25 \mathrm{~dB}$ HL at 1, 2, and $4 \mathrm{kHz}$. Furthermore, in some developed and developing countries screening intensity levels of 25, 30 and even $40 \mathrm{~dB}$ HL have typically been employed (AAA, 2011; Al-Rowaily, AlFayez, Aljomiey, AlBadr, \& Abolfotouh, 2012; Kam et al., 2013; Lo \& McPherson, 2013; Wu et al., 2014). A higher screening intensity level is sometimes used due to the presence of adverse background noise levels that are present in the test environment (Counter, 1986; Kam et al., 2013; McPherson, Law, \& Wong, 2010). The selected criterion for screening in turn has an effect on the referral rates, sensitivity and specificity of a screening programmes (Dodd-Murphy, Murphy, \& Bess, 2014). Ultimately these factors also determine the cost-effectiveness and feasibility of hearing screening programmes.

An immediate rescreen is an additional factor to consider for the purposes of reducing the referral rate. Screening is seen as a subjective test which requires the child to respond, thus external factors may influence the way a child may initially respond. Some of these external factors include the 
child's attention or understanding of instructions as well as the presence of environmental noise or distractions (Katz, 1994). American Speech-Language-Hearing Association (1997) and American Academy of Audiology (2011) guidelines indicate that an immediate rescreen should be conducted which includes removing the headphones from the child's head, repeating the instructions and carefully replacing the headphones over the ears. However, immediate rescreen results are often not reported in studies as a rescreen may not have been included (Kam et al., 2013; McPherson et al., 2010; Wu et al., 2014).

\subsection{Problem statement}

Referral rate is an essential consideration when determining the cost-effectiveness of a programme. As referral rates increase (as a result of a low screening intensity level) more follow-up diagnostic evaluations are required. An excessive referral rate will be prohibitive to the sustainability of screening programmes, especially in developing countries or under-resourced environments like those in the public school environment of South Africa. A higher screening intensity level can reduce the number of referrals, but may compromise the identification of milder hearing losses. This may be a trade-off that must be established by the constraints inherent to various contexts.

Less resourced contexts may require slightly higher hearing screening intensity levels to avoid overburdening health care systems that are already limited. In contrast more resourced countries may have lower screening intensity levels with higher referral rates with better sensitivity for identifying milder losses. Deciding on the appropriate screening intensity with regard to what the expected referral rate for school children will be, may assist in planning school-based programmes in different settings (McPherson, 2008).

\subsection{Objectives}

This study, therefore aims to investigate firstly the effect of screening intensity level and secondly the effect of an immediate rescreen on the referral rate in a school-based hearing screening programme.

\section{Material and methods}

\subsection{Design}

A within-subject study was conducted which consists of two phases. Phase one compared the referral rate at different screening intensity levels (20, 25 and $30 \mathrm{~dB}$ HL) whilst phase two determined the effect of an immediate rescreen in reducing the referral rate.

\subsection{Participants}

\subsubsection{Phase 1}

One hundred and thirty-five school-aged between the ages of 5 and 9 years (6.7 mean; 0.7 SD) participated in this phase of the study. The medium of instruction was English as all children's language of learning and teaching (LoLT) was English.

\subsubsection{Phase 2}

Three hundred and thirty-seven school-aged children between the ages of $5-10$ years (6.7 mean $\pm 1.09 \mathrm{SD}$ ) participated in Phase 2 of the study. The medium of instruction was also English since all the children's LoLT was English.

\subsection{Context}

Ethical clearance for the study was obtained from the University of Pretoria Institutional Review Board and the Gauteng Department of Education. Children were recruited from two local public schools in Tshwane, South Africa, with children from one school participating in Phase 1 and children from the other in Phase 2. All students, via their parents, in grade 1 to 3 in both schools were invited to participate. Only those children who provided signed assent along with a signed consent from their parent/caregiver participated in the study.

\subsection{Data collection procedures}

\subsubsection{Phase 1}

Screening was conducted over three days in a quiet room, provided by the local school. Ambient noise measurements could not be measured as the equipment was unavailable. An alternate approach, biologic noise level check, was conducted prior to the commencement of hearing screening. This has been defined as the ability to establish hearing thresholds at $10 \mathrm{~dB}$ HL below the screening level at all frequencies for a person with known normal hearing. If these thresholds could not be established, the area was not used (AAA, 2011). Audiology students from the University of Pretoria conducted the screenings under direct supervision.

Each subject received three hearing screenings at different screening intensity levels of 20, 25 and $30 \mathrm{~dB}$ HL respectively. The three screening levels were counterbalanced to minimize an order effect. Screening was conducted at 1, 2 and $4 \mathrm{kHz}$ as prescribed by current guidelines (AAA, 2011; ASHA, 1997). Left ears were tested first with an initial presentation at $1 \mathrm{kHz}$, $10 \mathrm{~dB}$ HL above the chosen screening level as a conditioning presentation. Test order was 1, 2 and then $4 \mathrm{kHz}$.

Children were instructed to raise their hand if they heard the sound with the screener sitting behind them (Fig. 1) administering the test. If a student did not respond to the sound at a specific frequency it was repeated once to confirm a no-response and then recorded as a refer result. A refer at any frequency in either ear constituted an overall referral.

\subsubsection{Phase 2}

Screening was conducted over five days with the same environmental conditions as used in Phase 1. Screening procedures where conducted at a screening intensity level of $25 \mathrm{~dB}$ HL at 1, 2 and $4 \mathrm{kHz}$. A refer at any frequency in either ear constituted an initial referral. A rescreen was done immediately following a refer result. This was done by removing the headphones and re-instructing the child. The screening audiometer on which the initial refer was recorded was used to conduct the rescreen. Diagnostic audiometry was then 


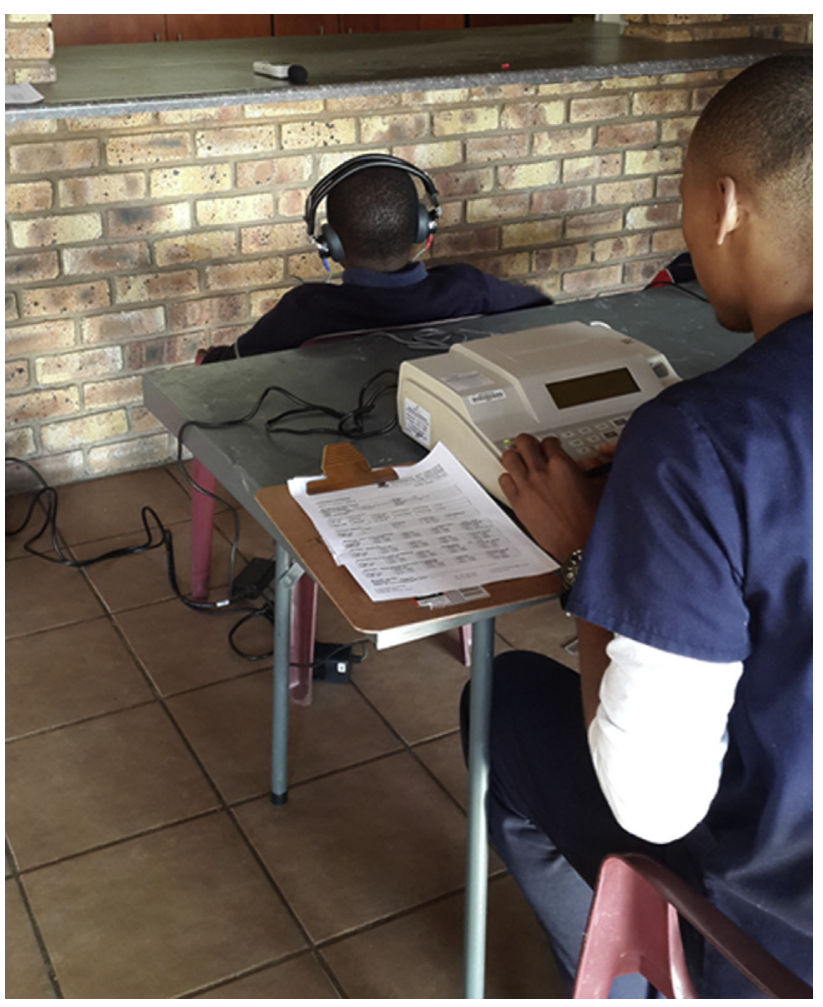

Fig. 1 - Screener administering hearing screening by sitting behind the patient.

conducted on children who referred on the rescreen. The school principal received hearing screening reports for all children tested. Based on the screening and diagnostic findings parents were provided with hearing screening reports and in the case of a referral, recommendations regarding follow-up assessments and interventions were made.

\subsection{Equipment}

\subsubsection{Phase 1}

Screening was conducted with one of two screening audiometers, a GSI Auto Tymp (Grayson Stadler, Eden Prairie, MN, USA) or an Interacoustics Impedance Audiometer AT 235 (William Demant, Smørum, Denmark), both using Telephonics TDH 39P headphones. Both instruments were calibrated according to ISO 389-1 specifications.

\subsubsection{Phase 2}

Screening was conducted with the same screening audiometers used in phase 1. Diagnostic pure tone air and bone conduction audiometry was conducted using the KUDUwave 5000 (GeoAxon, Pretoria, South Africa). This audiometer has been validated in a school-setting and previously described by Maclennan-Smith, Swanepoel, and Hall (2013). The KUDUwave is a Type 2 Clinical Audiometer (IEC 60645-1/2) controlled by software on a computer (Acer Travelmate 2492). The audiometer hardware is encased in circumaural earcups and powered by a USB cable plugged into the notebook. The transducers include embedded, custom insert earphones, which were covered by the circumaural cups after insertion. A response button was connected to the KUDUwave device to record patient responses to stimuli. The audiometer was calibrated prior to commencement of the study, insert earphones were calibrated in accordance with ISO 389-2 and the bone oscillator according to ISO 389-3.

\section{Calculation/data analysis}

\subsection{Phase 1}

Data analyses included cross-tabulations of referral rates at 20, 25 and $30 \mathrm{~dB}$ HL across 1,2 and $4 \mathrm{kHz}$ as well as overall cross tabulations for each ear at the above mentioned intensities. The McNemar test was performed to determine if there was a significant difference between referral rates amongst the three screening intensities (Statistical significance was noted as $\mathrm{p}<0.01)$. Data was analysed using SPSS (v22. Chicago, Illinois).

\section{2. $\quad$ Phase 2}

Data analyses included cross-tabulation of initial screening outcomes obtained at $25 \mathrm{~dB}$ HL compared to refer results obtained during the rescreen. Data was analysed using SPSSv22 (Chicago, Illinois).

\section{Results}

\subsection{Phase 1}

One hundred and thirty-five children were tested at the respective screening intensities. At $20 \mathrm{~dB}$ HL, ear specific referrals were most common at $2 \mathrm{kHz}$ (9.3\%) whilst at 25 and $30 \mathrm{~dB}$ HL referrals was at its highest at $4 \mathrm{kHz}$ (3.3\% and $1.9 \%)$ (Table 1). The referral rates obtained increased as the screening intensity level decreased across the frequencies 1, 2, $4 \mathrm{kHz}$.

A total of 23 children referred at $20 \mathrm{~dB} H \mathrm{HL}$, more than half $(14 / 23)$ of which passed at $25 \mathrm{~dB}$ HL, whilst only six $(6 / 14)$ referred at $30 \mathrm{~dB}$ HL. There was a significant difference between the referral rates obtained at screening intensity levels of 20 and $25 \mathrm{~dB}$ HL and 20 and $30 \mathrm{~dB}$ HL (McNemar, p < 0.01), but no significant differences between the referral rates at intensity levels 25 and $30 \mathrm{~dB}$ HL (Table 2).

Table 1 - Distribution of referrals across frequencies and different screening intensity levels ( $n=270$ ears).

\begin{tabular}{lccc} 
& $20 \mathrm{~dB}$ HL & $25 \mathrm{~dB}$ HL & $30 \mathrm{~dB}$ HL \\
\hline $1 \mathrm{kHz}$ & $5.9 \%$ & $1.5 \%$ & $1.5 \%$ \\
$2 \mathrm{kHz}$ & $9.3 \%$ & $1.9 \%$ & $0.7 \%$ \\
$4 \mathrm{kHz}$ & $6.3 \%$ & $3.3 \%$ & $1.9 \%$ \\
Referral rate per ear $^{\mathrm{a}}$ & $11.5 \%$ & $5.1 \%$ & $2.2 \%$ \\
\hline
\end{tabular}

${ }^{a}$ Number of referrals obtained for each ear $(n=270$ ears) across frequencies. 
Table 2 - Distribution of referrals at ear-specific screening intensity levels ( $n=135$ participants).

$20 \mathrm{~dB}$ HL $25 \mathrm{~dB} \mathrm{HL}$ $30 \mathrm{~dB} \mathrm{HL}$

\begin{tabular}{lccc}
\hline Right ear & $11.9 \%$ & $3.7 \%$ & $2.2 \%$ \\
Left ear & $11.1 \%$ & $6.7 \%$ & $2.2 \%$ \\
Referral rate per subject $^{\mathrm{a}}$ & $17 \%$ & $6.7 \%$ & $4.4 \%$ \\
\hline${ }^{a}{ }^{2}$ Number of referrals obtained across participants $(\mathrm{n}=135)$ and \\
frequencies.
\end{tabular}

\subsection{Phase 2}

The initial referral rate in this sample was $7.7 \%$ (Fig. 2) which reduced by one-third (2.7\%) with an immediate rescreen. Diagnostic audiometry conducted on all 17 students who failed the rescreen indicated that eight (47\%) were true positives presenting with a hearing loss in the referred ear/s. One of the 17 children was difficult to test and reliable results could not be obtained. Of these eight children, two presented with a unilateral mild-to-moderate conductive hearing loss, two with a unilateral moderate-to-profound mixed hearing loss, two with a bilateral mild-to-moderate sensorineural hearing loss, one with a bilateral profound sensorineural hearing loss and one with a bilateral asymmetrical moderate-to-profound mixed hearing loss in the right ear and a mild conductive hearing loss in the left ear.

\section{Discussion}

\subsection{Outline of results}

Despite widespread use of screening programmes to detect hearing loss, the recommended criterion for referral may not be ideal for resource-limited countries like South Africa (Kam et al., 2013). It has been proposed that the best screening programme is useless without definitive criterion for referral (Johnson, 1984). Programmes may be inappropriate or even unethical if there is not a sufficient audiological or medical infrastructure to cope with the possible cases of hearing loss identified through screening programmes (WHO, 1998). As a result implementing a school-based hearing screening

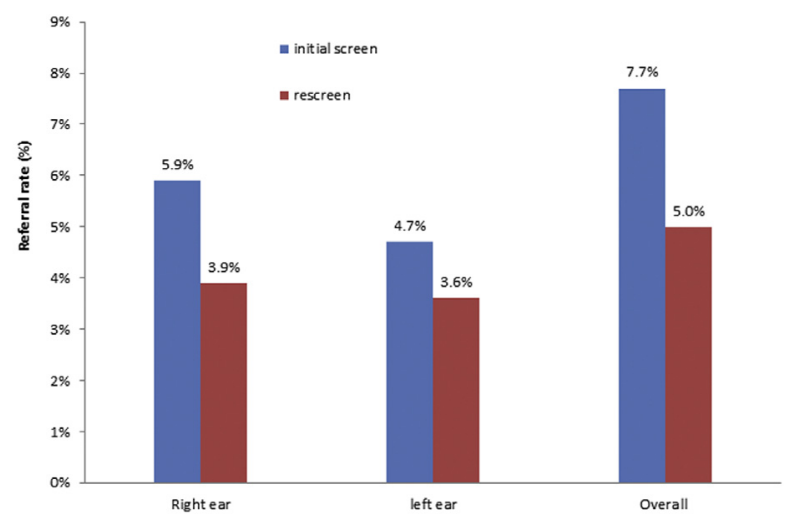

Fig. 2 - Distribution of referrals for initial screen and rescreen with screening intensity level at $25 \mathrm{~dB}$ HL across 1,2 and $4 \mathrm{kHz}$ ( $\mathrm{n}=337$ children). programme could become problematic if a large number or referrals could not be managed by a limited amount of followup resources, as is often is the case in developing countries (McPherson \& Olusanya, 2008).

A referral rate of $17 \%$ was obtained at $20 \mathrm{~dB}$ HL in the current study which means close to 1 in 5 children require following up services. These results were similar to the referral rate of $21.5 \%$ obtained by Sideris and Glattke (2006) who used the same referral criterion on a younger cohort of children between the ages of 2 and 5 years. These referral rates seem excessively high in comparison to those obtained at 25 and $30 \mathrm{~dB}$ HL (6.7\% and 4.4\% respectively). Dodd-Murphy et al. (2014) report similar findings using screening levels of 20 and $25 \mathrm{~dB} \mathrm{HL}$, on grade 2 learners, with referral rates of $17.8 \%$ and $7.6 \%$ respectively. Dodd-Murphy et al. (2014) confirmed that $20 \mathrm{~dB}$ HL is better suited to identify mild hearing losses but with referral rates 2.5 times higher. An excessive referral rate could be prohibitive to the sustainability of screening programmes, especially in developing countries or underresourced environments like South Africa. The use of a higher screening intensity level will reduce the number of referrals but will have less sensitivity for milder hearing losses. The ISHP (2012) specifies the use of $20 \mathrm{~dB}$ HL as the screening intensity level, however, a trade-off exists where less resourced countries like South Africa may require slightly higher screening intensity levels (e.g. $25 \mathrm{~dB}$ HL) to avoid overburdening health care systems that are already constrained. More resourced contexts that can deal with higher referral rates may however, opt for lower screening intensities (e.g. $20 \mathrm{~dB} \mathrm{HL}$ ) to improve identification of milder losses.

A screening intensity level of $25 \mathrm{~dB}$ HL was used in Phase 2 to determine the effect of an immediate rescreen on referral rates. The American Academy of Audiology (2011) and American Speech-Language-Hearing Association (1997) recommend a rescreen, but limited information has been reported on the effect of conducting an immediate rescreen (Lo \& McPherson, 2013; Szudek, Ostevik, Dziegielewski, RobinsonAnagor, \& Gomaa, 2012). Findings from the current study demonstrated that an immediate rescreen reduced the number of referrals initially obtained by $35 \%$. Furthermore it was noted that from the participants who referred the rescreen, nearly half (47\%) tested positive for some type of hearing loss after diagnostic testing.

\subsection{Practical implications}

As a result of the variability in the way school-based screening has been conducted there is no clear guideline on what the referral rate should ideally be. For newborn hearing screening (NHS) programmes, however, the recommended referral rate has been clearly prescribed to be less than $4 \%$ (Joint Committee on Infant Hearing, 2007). Referral rates obtained in this study at 25 and $30 \mathrm{~dB}$ HL were closer to these recommended rates from NHS programmes than the high referral rate obtained at $20 \mathrm{~dB}$ HL. Since no significant difference in referral rate was evident between 25 and $30 \mathrm{~dB}$ HL but a significant difference noted between 20 and $25 \mathrm{~dB}$ HL, a $25 \mathrm{~dB}$ HL screening intensity level may be most appropriate for resource-limited contexts. Employing $25 \mathrm{~dB}$ HL provides lower referral rates and is likely to have better sensitivity for milder 
hearing losses than a screening level of $30 \mathrm{~dB}$ HL. Furthermore, an immediate rescreen should be conducted on all children who referred on an initial screening to reduce the number of excessive referrals, so as to minimize the burden faced by follow-up services.

\section{Limitations of the study}

Limitations of the current study included the omission of an immediate rescreen during the first phase of the study to determine the reduction in referral rate at 20 and $30 \mathrm{~dB}$ HL. However, if a rescreen was conducted each child would receive more than six screens in total. This would have extended test time opening up the possibility of fatigue and a possible order effect. Additionally, all participants undertook three screening tests at different screening intensity levels which could have influenced the overall referral rates. The test sequence was however, counterbalanced to limit this effect. An additional limitation was that true sensitivity and the specificity of results could not be determined in either phases of the study because diagnostic testing was only conducted in the second phase for those children referring the screening. Furthermore, the study did not make use of acoustic immittance testing as a secondary screening (AAA, 2011) to identify or rule out the presence of any middle ear pathologies. However, diagnostic testing was conducted which determined the presence of conductive or mixed hearing losses on the children who did refer on the rescreen in phase 2 of the study.

\section{Conclusion}

In resource-limited contexts, a screen intensity level of $25 \mathrm{~dB}$ $\mathrm{HL}$ with an immediate rescreen at recommended frequencies (1, 2 and $4 \mathrm{kHz}$ ) can significantly reduce the overall referral rate to avoid overburdening health care resources. Of those children referred for follow-up services using this protocol close to half may be expected to present with hearing loss. It is recommended that a follow-up study be conducted to evaluate the follow-up services and the referral pathways available in resource-limited countries like South Africa.

\section{Conflict of interest}

The authors declare no conflict of interests.

\section{Acknowledgements}

We thank the Audiology students at the University of Pretoria for assisting with the data collection as well as the public schools and their students for participating in this study. Furthermore, the financial assistance of the National Research Foundation (NRF) towards this research is hereby acknowledged. Opinions expressed and conclusions arrived at, are those of the author and are not necessarily to be attributed to the NRF.

\section{REFERENCES}

Al-Rowaily, M. A., AlFayez, A. I., AlJomiey, M. S., AlBadr, A. M., \& Abolfotouh, M. A. (2012). Hearing impairments among Saudi preschool children. International Journal of Pediatric Otorhinolaryngology, 76(11), 1674-1677.

American Academy of Audiology (AAA). (2011). Clinical practice guidelines childhood hearing screening. Specifications for audiometers. Retrieved from: http://www.cdc.gov/ncbddd/ hearingloss/documents/aaa_childhood-hearingguidelines 2011.pdf.

American Speech-Language-Hearing Association. (1997). Guidelines for audiologic screening. Retrieved from: http://www. asha.org/policy/GL1997-00199/.

Bamford, J., Fortnum, H., Bristow, K., Smith, J., Vamvakas, G., Davies, L., et al. (2007). Current practice, accuracy, effectiveness and cost-effectiveness of the school entry hearing screen. Health Technology Assessment (Winchester, England), 11, 1-168.

Bess, F. H., \& Dodd-Murphy, J. D. (1998). Children with minimal sensorineural hearing loss: prevalence, educational performance, and functional health status. Ear and Hearing, 17 $1-11$.

Counter, S. A. (1986). Audiological screening of Amerindians of the Surinam rainforest. Scandinavian Audiology, 15, 57-64.

Cunningham, M., \& Cox, E. O. (2003). Hearing assessment in infants and children: recommendations beyond neonatal screening. American Academy of Pediatrics, 111(2), 436-440.

Davis, J. M., Elfenbein, J., Schum, R., \& Bentler, R. A. (1986). Effects of mild and moderate hearing impairments on language, educational, and psychosocial behavior of children. Journal of Speech and Hearing Disorders, 51(1), 53.

Dodd-Murphy, J., Murphy, W., \& Bess, F. H. (2014). School screenings and identification of mild sensorineural hearing loss. American Journal of Audiology, 23(4), 365-373.

Fortnum, H. (2003). Epidemiology of permanent childhood hearing impairment: implications for neonatal hearing screening. Audiological Medicine, 1(3), 155-164.

Grote, J. (2000). Neonatal screening for hearing impairment. Lancet, 355(9203), 513-514.

Integrated School Health Policy (ISHP). (2012). Department of Basic Education and Department of Health. Pretoria, South Africa.

Johnson, D. W. (1984). Screening, search, identification: strategies for referral. In Diagnosis and management of hearing loss (pp. 153-171).

Joint Committee on Infant Hearing. (2007). Year 2007 position statement: principles and guidelines for early hearing detection and intervention programs. Pediatrics, 120, 898-921.

Kam, A. C. S., Gao, H., Li, L. K. C., Zhao, H., Qiu, S., \& Tong, M. C. F. (2013). Automated hearing screening for children: a pilot study in China. International Journal of Audiology, 52(12), 855-860.

Katz, J. (1994). Handbook of clinical audiology (4th ed.). Lippincott Williams \& Wilkins.

Lo, A. H. C., \& McPherson, B. (2013). Hearing screening for school children: utility of noise-cancelling headphones. International Journal of Pediatric Otorhinolaryngology, 50(2), 93-98.

Lopez, A. D., Mathers, C. D., Ezzati, M., Jamison, D. T., \& Murray, C. J. L. (2006). Global and regional burden of disease and risk factors, 2001: systematic analysis of population health data. Lancet, 367(9524), 1747-1757.

Lü, J., Huang, Z., Yang, T., Li, Y., Mei, L., Xiang, M., et al. (2011). Screening for delayed-onset hearing loss in preschool children 
who previously passed the newborn hearing screening. International Journal of Pediatric Otorhinolaryngology, 75(8), 1045-1049.

Maclennan-Smith, F., Swanepoel, D. W., \& Hall, J. (2013). Validity of diagnostic pure-tone audiometry without a sound-treated environment in older adults, (October 2012) (pp. 66-73).

McKay, S., Gravel, J. S., \& Tharpe, A. M. (2008). Amplification considerations for children with minimal or mild bilateral hearing loss and unilateral hearing loss. Trends in Amplification, 12(1), 43-54.

McPherson, B. (2008). Audiology in developing countries. New York: Nova Science Publishers, Inc.

McPherson, B., Law, M. M. S., \& Wong, M. S. M. (2010). Hearing screening for school children: comparison of low-cost, computer-based and conventional audiometry. Child: Care, Health and Development, 36(3), 323-331.

Meyer, M., Swanepoel, D. W., Van Der Linda, M., \& Le Roux, T. (2012). Early detection of infant hearing loss in the private health care sector of South Africa. International Journal of Pediatric Otorhinolaryngology, 76, 698-703.

Muse, C., Harrison, J., Yoshinaga-Itano, C., Grimes, A., Brookhouser, P. E., Epstein, S., et al. (2013). Supplement to the JCIH 2007 position statement: principles and guidelines for early intervention after confirmation that a child is deaf or hard of hearing. Pediatrics, 131(4), 1324-1349.

Sideris, I., \& Glattke, T. J. (2006). A comparison of two methods of hearing screening in the preschool population. Journal of Communication Disorders, 39(6), 391-401.

Skarzynski, H., \& Piotrowska, A. (2012). Screening for pre-school and school-age hearing problems: European Consensus
Statement. International Journal of Pediatric Otorhinolaryngology, 76, 120-121.

Smith, R. J. H., Bale, J. F., \& White, K. R. (2005). Sensorineural hearing loss in children. Lancet, 365(9462), 879-890.

Szudek, J., Ostevik, A., Dziegielewski, P., Robinson-Anagor, J., \& Gomaa, N. (2012). Can u hear me? Validation of an iPod-based hearing loss screening test. Journal of Otolaryngol Head Neck Surg, 41, 78-84.

Tharpe, A. M. (2008). Trends in amplification. Trends in Amplification, 12(1), 7-15.

Theunissen, M., \& Swanepoel, D. W. (2008). Early hearing detection and intervention services in the public health sector in South Africa. International Journal of Audiology, 47, 23-29.

White, K. (2010). Twenty years of early hearing detection and intervention (EHDI): Where we've been and what we've learned. ASHA Audiology Virtual Conference. ASHA Audiology Virtual Conference.

Word Health Organisation. (1998). Hearing aid services - Needs and technology assessment for developing countries. Report of a WHO/ CBM workshop. Bensheim, Germany.

World Health Organisation. (2014). The global burden of disabling hearing impairment: A call to action, 92 pp. 367-373).

World Health Organization. (2013). WHO | Estimates. Retrieved from. World Health Organization http://www.who.int/pbd/ deafness/estimates/en/index.html.

Wu, W., Lü, J., Li, Y., Kam, A. C. S., Fai Tong, M. C., Huang, Z., et al. (2014). A new hearing screening system for preschool children. International Journal of Pediatric Otorhinolaryngology, 78(2), 290-295. 